\title{
Synthesis of Oligonucleotide Conjugates Carrying Viologen and Fluores- cent Compounds
}

\author{
Margarita Alvira ${ }^{1}$, Susan J. Quinn ${ }^{2}$, Anna Aviñó ${ }^{1}$, Donald Fitzmaurice ${ }^{3}$ and Ramon Eritja*,1 \\ ${ }^{I}$ Institute for Research in Biomedicine (IRB Barcelona), Institute for Advanced Chemistry of Catalonia (IQAC), Spanish \\ Research Council (CSIC), Networking Centre on Bioengineering, Biomaterials and Nanomedicine (CIBER-BBN), Josep \\ Samitier 1-5, E-08028 Barcelona, Spain \\ ${ }^{2}$ School of Chemistry, Trinity College Dublin, University of Dublin, Dublin 2, Ireland \\ ${ }^{3}$ School of Chemistry and Chemical Biology, University College Dublin, Dublin 4, Ireland
}

\begin{abstract}
The preparation of oligonucleotide conjugates carrying viologen and fluorescein is described. Reaction of the appropriate carboxyl derivatives with oligonucleotides carrying aliphatic amino groups gave the desired compounds. A simple method for the introduction of the amino group at the 5'-end of the oligonucleotides is reported.
\end{abstract}

\section{INTRODUCTION}

The conjugation of oligonucleotides to different functional molecules is a valuable tool that allows for modulation of both therapeutical and biotechnological properties. Such conjugate groups confer a variety of important properties, these include fluorescent emission to allow sensitive detection $[1,2]$, altered hydrophobicity or bioaffinity $[3,4]$, enhanced cellular uptake $[5,6]$, resistance towards degradation [7], novel electrochemical properties [8] and the ability to coordinate metal ions [9], to mention just a few. Accordingly, convenient synthetic protocols for the preparation of oligonucleotide conjugates are of great interest.

The two principle methods for the synthesis of oligonucleotide conjugates involve (1) the introduction of the modification as an individual phosphoramidite or (2) postsynthetic modification of oligonucleotide through the coupling of a ligand once the oligomer is fully synthesized. The main disadvantage of the first method is the requirement that each conjugate molecule must first be prepared as a phosphitylated building block. For this reason the second approach is considered the more versatile strategy and it typically involves the insertion of a masked nucleophilic or electrophilic group into the oligonucleotide chain. To date, aliphatic amino groups have been extensively used for this purpose. Attachment of this group to one end of the oligonucleotide allows for facile reaction with incoming functional groups in the form of electrophiles (e.g., activated carboxylic groups [10-12], isothiocyanates [13, 14], alkylating agents and aldehydes with further reduction by sodium borohydrate $[15,16])$.

In most cases the desired functional molecule is introduced during solid-phase synthesis. However, this is not

\footnotetext{
*Address correspondence to this author at the Institute for Research in Biomedicine (IRB Barcelona), Institute for Advanced Chemistry of Catalonia (IQAC), Spanish Research Council (CSIC), Networking Centre on Bioengineering, Biomaterials and Nanomedicine (CIBER-BBN), Josep Samitier 15, E-08028 Barcelona, Spain; E-mail: recgma@cid.csic.es
}

possible in instances where the desired ligand to be attached is incompatible with conditions under which oligonucleotide deprotection and cleavage occurs. This is the case for base labile compounds (such as the viologen molecule reported in this article). These base labile groups necessitate the use of appropriately modified linkers that allow for the oligonucleotide synthesis to take place on the support and conjugation to occur after the cleavage step.

We are interested in the preparation of oligonucleotide conjugates carrying compounds such as viologens, and fluorescent compounds such as fluorescein. Viologens $(1,1-$ disubstituted 4,4'-bipyridinium dications) are a well-studied class of redox chromophores [17-21]. They have one of the lowest redox potentials of any organic molecule [17]. In addition, they exhibit a large change in the visible extinction coefficient following reduction and possess a first reduction potential that is essentially $\mathrm{pH}$ independent. Due to these properties many attempts have been made to incorporate these molecules in electrochromic devices [20-22]. Moreover these molecules may be used as electron acceptors to measure electron transport in DNA [22, 23]. To this end, viologens have to be incorporated in synthetic oligonucleotides but preliminary studies have shown that these molecules are not stable to the ammonia [23-25], reagent that is used for the removal of the protecting groups in DNA synthesis. In the present report we describe the efficient synthesis of oligonucleotide conjugates by reaction of oligonucleotides carrying amino groups with the appropriate carboxyl derivatives.

A protected aliphatic amino group can be readily introduced in the 5 ' terminus through commercially available phosphoramidites, such as the $\mathrm{N}$-monomethoxytrityl (MMT) -6-amino-hexyl phosphoramidite [26]. This compound was used to obtain the 5'-amino sequences that were conjugated to viologen in this study.

However previous studies [27] have shown that MMT protection does not completely prevent the reactivity of the 5 '-amino group toward $\mathrm{N}$-acetylation (coming from capping 

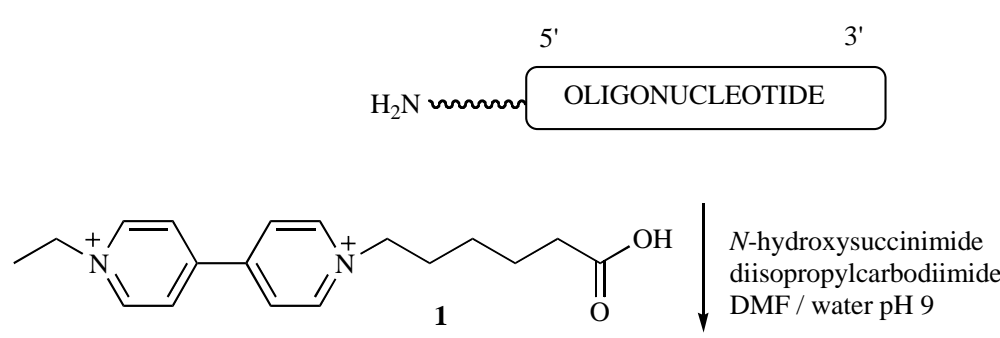

$2 \mathrm{PF}_{6}^{-}$

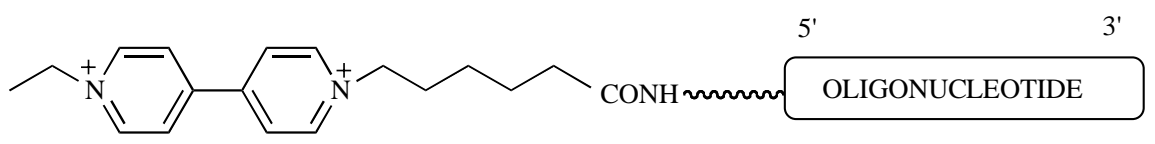

Scheme 1. Synthesis of oligonucleotide-viologen conjugates.

reagents). This effect was also observed by Zaramella et al. [28] during the assembly of a peptide at the 5'-end of the target oligonucleotide. N-acetylation of the amino group leads to a loss in reactivity and consequently reduces the efficiency of oligonucleotide functionalisation. Furthermore, the deprotection of MMT occurs under stringent acid conditions and typically involves reaction of the oligonucleotide for over one hour in $80 \%$ aqueous acetic acid solution. In some cases this does not remove MMT completely [29, 30], and can cause depurination. Alternatively, in a reverse of this strategy, chloroacetamidohexyl phosphoramidite has been used to introduce an electrophillic group in oligonucleotides [31]. In this case the reaction of primary amines or mercaptans with this chloroacetamido group afforded a variety of oligonucleotide conjugates in good yields.

In the work presented here we have overcome the challenges mentioned above by developing a protocol that employs a 6-bromohexyl phosphoramidite intermediate that leads to a 5'-aminohexyl oligonucleotide after ammonia cleavage from the support.

\section{RESULTS AND DISCUSSION}

\section{Synthesis of Oligonucleotides Carrying Viologen}

The viologen derivative, 1-Ethyl-1'-(5-carboxypentyl)4,4-bipyridinium di(hexafluorophosphate), 1 carrying a carboxylic group was chosen for reaction with the aminooligonucleotides. This compound was prepared according to literature procedures [32-34].

The stability of compound 1 to ammonia was checked by treating aliquots of compound with concentrated ammonia. The rapid decomposition of viologen 1 was observed, even at room temperature. The decomposition was revealed by HPLC analysis with the initial compound replaced by two new products with higher retention times. These two compounds are most probably the olefins from a $\beta$-elimination reaction of the $\mathrm{N}$-alkyl quaternary ammonium groups. These results are in agreement with previous studies [22, 23] and suggest that viologen molecules should be incorporated to oligonucleotides after ammonia treatment (Scheme 1).

Oligonucleotides A $\left(5^{\prime}-\mathrm{NH}_{2}\right.$-TCAGTAGTCAG) and B (5'- $\mathrm{NH}_{2}$-TTCAGTAGTCAG) with a primary amino group attached to the 5'-end were synthesized using the $N$-MMT-6aminohexyl phosphoramidite [26]. 5'-Amino oligonucleotides were treated with viologen 1 using $\mathrm{N}$ - hydroxysuccinimide and diisopropylcarbodiimide as activating agents. The resulting products were desalted with a Sephadex G-25 column and purified by RP-HPLC (Table 1). The HPLC analysis revealed a major peak which was collected. Mass spectrometry confirmed that the desired product corresponded to the major peak on the chromatogram (Table 1). In both cases, the conversion to the desired compound was greater than $80 \%$.

\section{Synthesis of Oligonucleotides Carrying Amino Groups at the 5'-end Using the Bromohexyl Phosphoramidite}

An alternative synthesis of the oligonucleotide carrying aminohexyl group was achieved following the steps presented in Scheme 2. Oligonucleotide sequences C (5'CTTCCTCCTCT-3') and D (5'-GACTCGAT-3') were synthesized using standard phosphoramidites. The bromohexyl linker was incorporated into the 5 ' terminus through a phosphoramidite that was obtained from 6-bromohexan-1-ol according to the protocol described in [35].

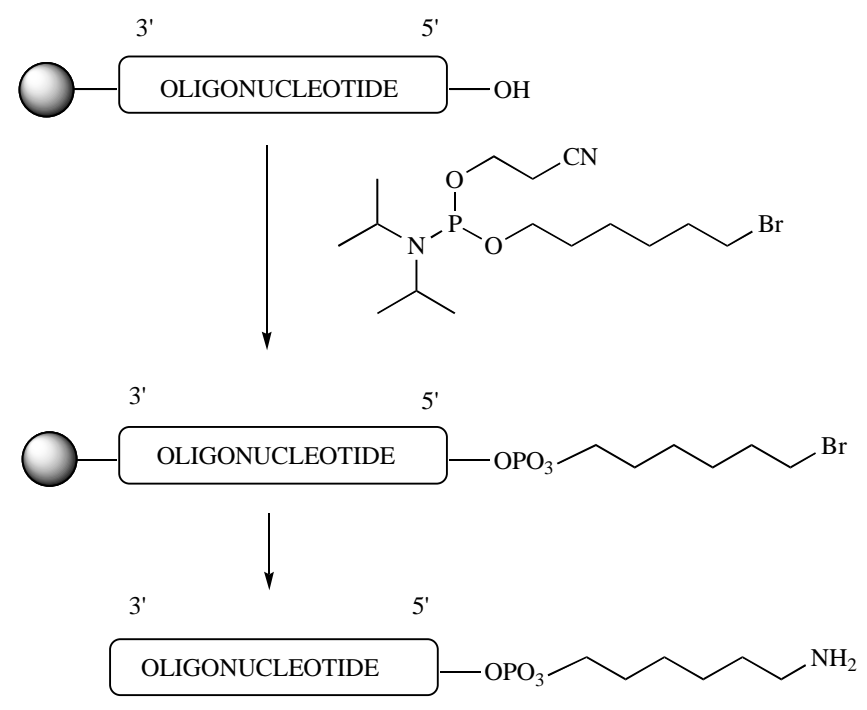

Scheme 2. Synthesis of the 5'-aminohexyl oligonucleotides.

The supports carrying the 5'-Br-oligonucleotide were treated with concentrated aqueous ammonia at room temperature, giving the desired 5'-aminohexyl-oligonucleotide as the major product (see chromatogram in Fig. 1). The 5'bromohexyl oligonucleotide was also observed. Various attempts to enhance the yield of nucleophilic substitution were 
made using longer times and higher temperature but only a slight improvement was observed. (Fig. 2). The purified oligonucleotides had the expected mass.

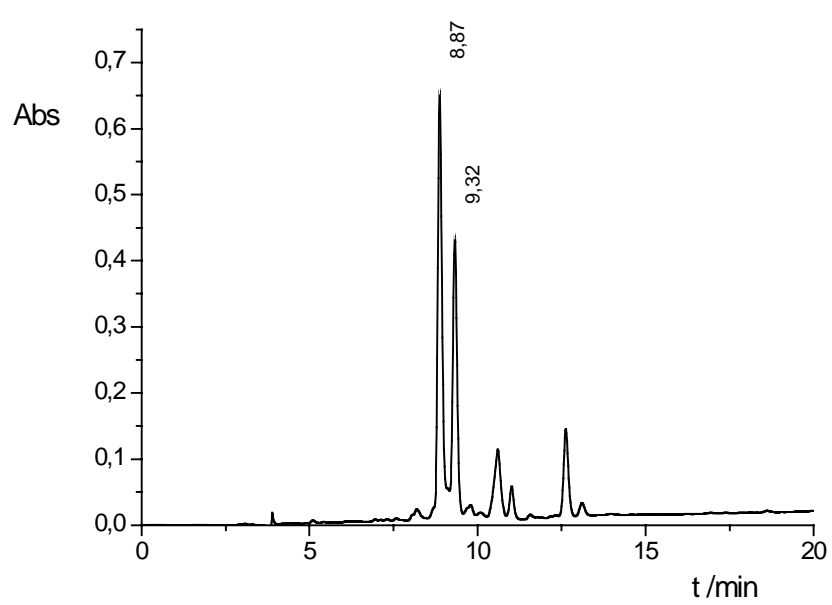

Fig. (1). HPLC profile obtained after treatment of the 5'-Broligonucleotide-support (sequence $\mathrm{C}$ ) with concentrated aqueous ammonia for 3 hours at room temperature.

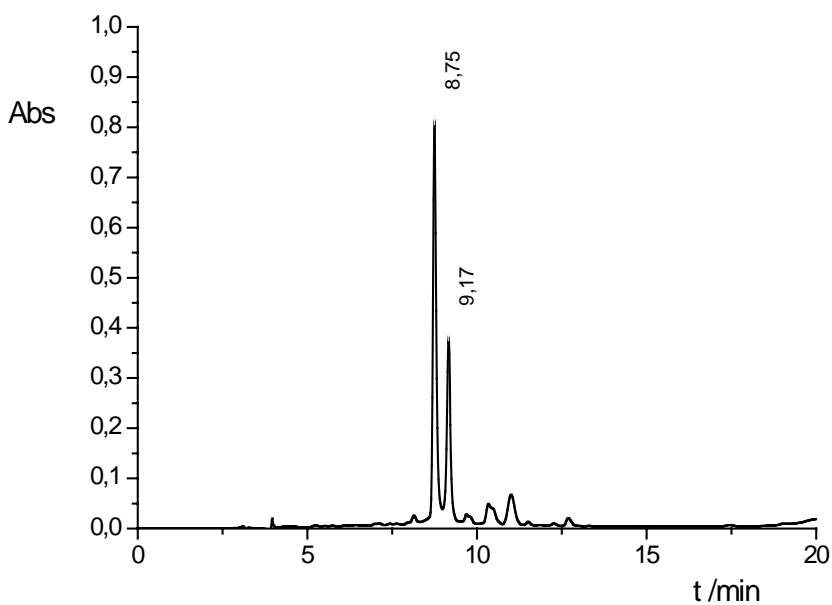

Fig. (2). HPLC profile obtained after treatment of the 5'-Broligonucleotide-support (sequence $\mathrm{C}$ ) with concentrated aqueous ammonia for 5 hours at $55^{\circ} \mathrm{C}$.

To confirm that our purified products contained the amino terminal group and that the obtained mass does not come from a substitution of bromide with an hydroxyl group (in which case the oligomer would be expected to have a similar mass) we decided to couple a ligand with a carboxylic group that would lead to an easily detectable conjugate.

5(6)-carboxyfluorescein N-hydroxysuccinimide ester 2 was coupled to the oligonucleotide $\mathrm{C}$ as detailed in the Experimental Section. Fig. (3) shows the HPLC profile of the reaction. The desired fluorescein labelled oligonucleotide was obtained as a major product ( $\mathrm{tR}=11.2 \mathrm{~min})$. The peak eluting at $10.2 \mathrm{~min}$ corresponds to the coupling of the oligomer with one of the isomers of the fluorescein (as the commercial reagent is a mixture of 5- and 6carboxyfluorescein isomers). Both products had the expected mass.

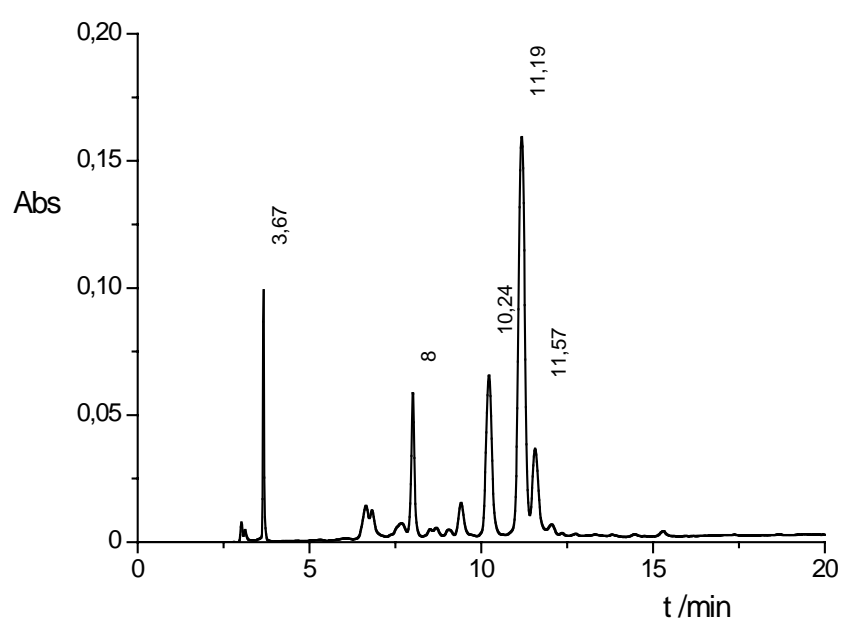

Fig. (3). HPLC profile obtained after conjugation of the $5^{\prime}-\mathrm{NH}_{2}-$ oligonucleotide (sequence C) with the fluorescein derivative 2 .

\section{CONCLUSIONS}

In conclusion, we have demonstrated that viologen modified oligonucleotides can be prepared by post-synthetic conjugation of oligonucleotides carrying amino groups. This is relevant as the introduction of these molecules in DNA is of interest for studies DNA-mediated electron transfer. We also described a straightforward protocol to incorporate an aminohexyl linker at the 5' terminus of an oligonucleotide that allows the attachment of appropriately functionalised ligands in solution phase. Finally, the use of a bromohexyl linker as an intermediate for the introduction of the nucleophilic amino group was shown to overcome the disadvantages presented by some protected amino modifier phosphoramidites. The desired 5'-amino oligonucleotide was easily obtained upon addition of ammonia during the cleavage of the product from the solid support. We believe that these observations present an important contribution to this growing field.

\section{EXPERIMENTAL SECTION}

\section{Oligonucleotide Synthesis and Purification}

Oligonucleotide sequences were prepared using solidphase methodology and 2-cyanoethyl phosphoramidites as monomers. The syntheses were performed on an Applied Biosystems Model 3400 DNA synthesizer using $1 \mu \mathrm{mol}$ scale. Oligonucleotides were purified by reversed-phase HPLC. HPLC conditions I: a 20 min gradient from $0 \% \mathrm{~B}$ to $50 \%$ B; II; using a 20 min linear gradient from 5 to $35 \%$ of $B$ with a flow rate of $3 \mathrm{ml} / \mathrm{min}$. HPLC solutions are as follows: Solvent A: $5 \% \mathrm{ACN}$ in $100 \mathrm{mM}$ triethylammonium acetate (pH 6.5) and solvent B: $70 \% \mathrm{ACN}$ in $100 \mathrm{mM}$ triethylammonium acetate $\mathrm{pH}$ 6.5. The column used in conditions I was PRP-1, $10 \mu \mathrm{m}$, (Hamilton), 250x8 mm. In conditions II was a Nucleosil $120 \mathrm{C} 18(10 \mu \mathrm{m}), 200 \times 10 \mathrm{~mm}$.

\section{Stability of Compound 1 in Ammonia}

1-mg aliquots of compound $\mathbf{1}$ were dissolved in concentrated ammonia $(1 \mathrm{ml})$. The samples were treated at room temperature and $55^{\circ} \mathrm{C}$. Then samples were concentrated and analyzed by HPLC (conditions I). 
Table 1 Oligonucleotide Conjugates Prepared in this Work

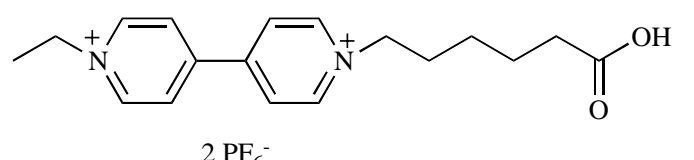

1<smiles>O=C(O)c1ccc(-c2c3ccc(=O)cc-3oc3cc(O)ccc23)cc1</smiles>

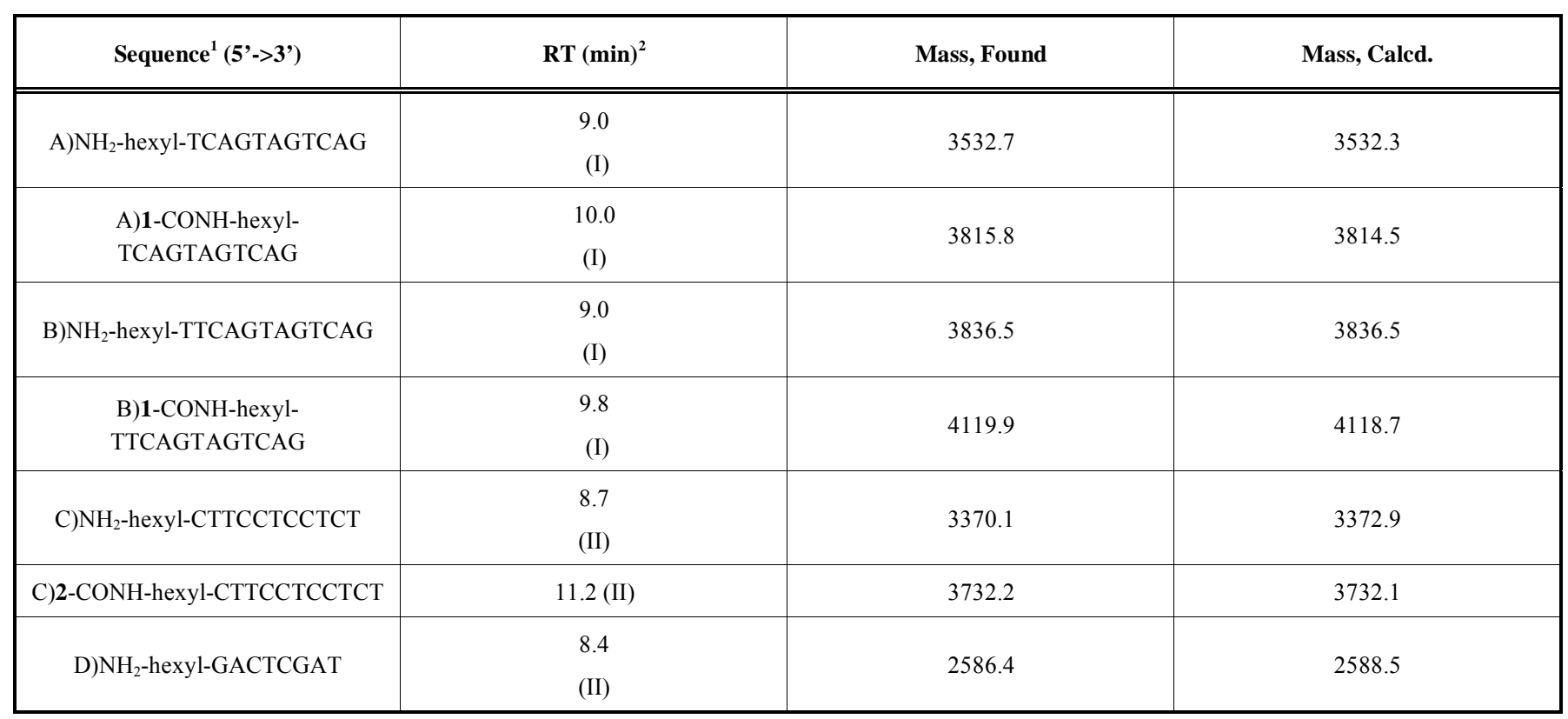

${ }^{1}$ 1: viologen derivative . 2: fluoresceine derivative.

${ }^{2}$ HPLC conditions. I: a 20 min gradient from $0 \%$ B to $50 \%$ B. II: a 20 min gradient from $5 \%$ B to $35 \%$ B (a more detailed description in experimental section).

\section{Synthesis of Oligonucleotide Carrying Viologens}

Oligonucleotides A (5'- $\mathrm{NH}_{2}$-TCAGTAGTCAG) and B (5'-NH ${ }_{2}$-TTCAGTAGTCAG) with a primary amino group (N-MMT-6-aminohexanol phosphoramidite) attached to the 5 '-end were synthesized on an automatic DNA synthesizer using the standard phosphoramidites. They were deprotected with ammonia and desalted with a Sephadex G-25 column (NAP-10). Interfering ammonium ions present from the deprotection steps were removed by passing a solution of the oligonucleotide in water through a small column filled with Dowex $50 \times 2$ (sodium form).

5'-Amino oligonucleotides (30 O.D. units) were dissolved in $0.2 \mathrm{ml}$ of $0.5 \mathrm{M}$ aqueous sodium carbonate buffer

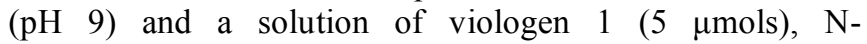
hydroxysuccinimide ( $5 \mu \mathrm{mols})$ and diisopropylcarbodiimide ( $5 \mu$ mols) in $0.15 \mathrm{ml}$ of N,N-dimethylformamide was added. The mixtures were incubated at room temperature overnight and the solutions were concentrated to dryness. The resulting products were first desalted with a Sephadex G-25 column (NAP-10) and purified by RP-HPLC (Table 1). The HPLC analysis revealed a major peak which was collected. Mass spectrometry confirmed that the desired product corresponded to the major peak on the chromatogram (Table 1). In both cases, the conversion to the desired compound, estimated by comparison of the areas under the peaks, was higher than $80 \%$. Recoveries were 9 OD (30\%) for sequence $\mathrm{A}$ and $15 \mathrm{OD}(50 \%)$ for sequence $\mathrm{B}$.

\section{Synthesis of the Phosphoramidite of 6-Bromohexanol}

6-Bromohexanol was reacted with 2-cyanoethoxy- $N, N$ diisopropylaminochlorophosphine in dry acetonitrile and $\mathrm{N}, \mathrm{N}$-diisopropylethylamine as described in reference [35].

\section{Synthesis of 5'-Amino-Oligonucleotides Using the Phos- phoramidite of 6-Bromohexanol}

Sequences C: 5'-CTTCCTCCTCT-3' and D: 5'GACTCGAT-3' were prepared in an automatic DNA synthesizer. $\mathrm{A}$ and $\mathrm{C}$ nucleobases were protected with the benzoyl group. G was protected either with the classical isobutyryl group or with the more base-labile dimethylformamidine group. The phosphoramidite of 6-bromohexanol was coupled in the last cycle (without performing final TCA detrytilation step) for the later introduction of the amino group at the 5'-end. The resulting 5'-bromohexyl oligonucleotides were treated with concentrated aqueous ammonia (Fig. 1). The resulting 5'-amino oligonucleotides were purified by reverse-phase HPLC (conditions II). The two main peaks were collected, desalted with a Sephadex G-25 column and analyzed by MALDI-TOF. In all the cases the first eluted product corresponds to the desired 5'-aminohexyl oligonucleotide (MALDI-MS of oligonucleotide $\mathrm{C}\left[\mathrm{M}-\mathrm{H}^{+}\right]$: 3370.1 , expected for $\mathrm{C}_{110} \mathrm{H}_{152} \mathrm{~N}_{29} \mathrm{O}_{72} \mathrm{P}_{11}$ : 3372.8.; MALDI- 
expected for $\mathrm{C}_{110} \mathrm{H}_{152} \mathrm{~N}_{29} \mathrm{O}_{72} \mathrm{P}_{11}$ : 3372.8.; MALDI-MS of oligonucleotide D $\left[\mathrm{M}-\mathrm{H}^{+}\right]$: 2586.4, expected for $\mathrm{C}_{84} \mathrm{H}_{113} \mathrm{~N}_{31} \mathrm{O}_{49} \mathrm{P}_{8}: 2588.5$ ) while the second one is the nonreacted 5'-bromohexyl oligonucleotide (MALDI-MS of oligonucleotide $\mathrm{C}\left[\mathrm{M}-\mathrm{H}^{+}\right]$: 3435.0, expected for $\mathrm{C}_{110} \mathrm{BrH}_{150} \mathrm{~N}_{28} \mathrm{O}_{72} \mathrm{P}_{11}$ : 3436.7; MALDI-MS of oligonucleotide $\mathrm{D}\left[\mathrm{M}-\mathrm{H}^{+}\right]: 2651.3$, expected for $\mathrm{C}_{84} \mathrm{BrH}_{111} \mathrm{~N}_{30} \mathrm{O}_{49} \mathrm{P}_{8}$ : 2652.3).

\section{Fluorescein Conjugation to Oligonucleotides}

A solution of the deprotected oligonucleotide $\mathrm{C}$ was passed through a column filled with Dowex $50 \times 2$, sodium form, to remove the interfering ammonium ions coming from the cleavage step. $50 \mathrm{nmol}$ of 5 '-amino oligonucleotides (5.5 O.D units) were dissolved in $0.2 \mathrm{ml}$ of $0.5 \mathrm{M}$

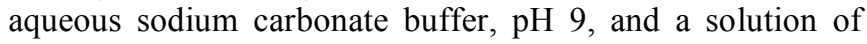
5(6)-carboxyfluorescein N-hydroxysuccinimide ester (10 $\mu \mathrm{mol})$ in $50 \mu \mathrm{l}$ of dioxane was added. The mixture was incubated at room temperature overnight and subsequently concentrated to dryness. The resulting products were first desalted with a Sephadex G-25 column and purified by RPHPLC. The desired oligonucleotide eluted at $11.2 \mathrm{~min}$ as a major peak, using the eluting conditions mentioned above. MALDI-MS: 3732.2, (expected for $\mathrm{C}_{131} \mathrm{H}_{163} \mathrm{~N}_{29} \mathrm{O}_{78} \mathrm{P}_{11}$ : 3732.1).

\section{ACKNOWLEDGEMENTS}

This work was supported by the European Communities (NANO-3D, NMP4-CT2005-014006; DYNAMO, NESTADV028669) by the Spanish Ministry of Education (grants NAN2004-09415-C05-03, BFU2004-02048), and the Generalitat de Catalunya (2005/SGR/00693). M.A. thanks the Spanish Ministry of Education for the predoctoral fellowship.

\section{REFERENCES}

[1] Vinayak, R. Tetrahedron Lett., 1999, 40, 7611.

[2] Wang, L.; Reipa, V.; Blasic, J. Bioconjugate Chem., 2004, 15, 409.

[3] Asseline, U.; Delarue, M.; Lancelot, G.; Toulmé, F.; Thuong, N.T.; Montenay-Garestier, T.; Hélène, C. Proc. Natl. Acad. Sci. U.S.A., 1984, 81, 3297.

[4] Martyanov, I.V.; Zakharova, O.D.; Sottofattori, E.; Pyshnyi, D.V.; Yurchenko, E.Y.; Babbi, P.; Mazzei, M.; Balbi, A.; Andreola, M.L.; Litvak, S.; Tarrago-Litvak, L.; Nevinsky, G.A. Antisense Nucleic Acid Drug Dev., 1999, 5, 473.

[5] Moulton, H.M.; Nelson, M.H.; Hatlevig, S.A.; Reddy, M.T.; Iversen, P.L. Bioconjug. Chem., 2004, 15, 290.
[6] Ye, Z.; Cheng, K.; Guntaka, R.V.; Mahato, R.I. Bioconjug. Chem., 2006, 17, 823 .

[7] Manoharan, M. Antisense Nucleic Acid Drug Dev., 2002, 12, 103

[8] Korri-Youssoufi, H.; Yassar, A. Biomacromolecules, 2001, 2, 58.

[9] Mestre, B.; Pitié, M.; Loup, C.; Claparols, C.; Pratviel, G.; Meunier, B. Nucleic Acids Res., 1997, 25, 1022.

[10] Milesi, D.; Kutyavin, I.; Lukhtanov, E.A.; Gorn, V.V.; Reed, M.W. Methods Enzymol., 2000, 313, 164.

[11] Bologna, J-C.; Imbach, J-L.; Morvan, F. Tetrahedron Lett., 2000, $41,7317$.

[12] Frieden, M.; Aviñó, A.; Tarrasón, G.; Escorihuela, M.; Piulats, J.; Eritja, R. Chem. Biodivers., 2004, 1, 930.

[13] Anno, Y.; Kubo, T.; Ueki, R.; Yano, M.; Sasaki, K.; Ohba, H.; Fujii, M. Nucleosides Nucleotides Nucleic Acids, 2003, 22, 1451.

[14] Sobkowski, M.; Kraszewski, A.; Stawinski, J. Nucleosides Nucleotides, 1998, 17, 253.

[15] Delair, T.; Badey, B.; Domard, A.; Pichot, C.; Mandrand, B. Polym. Adv. Technol., 1997, 8, 297.

[16] Defrancq, E.; Hoang, A.; Vinet, F.; Dumya, P. Bioorg. Med. Chem. Lett., 2003, 13, 2683.

[17] Bird, C.L.; Kuhn, A.T. Chem. Soc. Rev., 1981, 10, 49

[18] Watanabe, T.; Honda, K. J. Phys. Chem., 1982, 86, 2617.

[19] Marguerettaz, X., O'Neil, R., Fitzmaurice, D. J. Am. Chem. Soc., 1994, 116, 2629.

[20] Slama-Schwok, A., Ottolenghi, M., Avnir, D. Nature, 1992, 355, 240.

[21] Cinnsealach, R., Boschloo, G., Rao, S.N., Fitzmaurice, D. Sol. Energy Mater. Sol. Cells, 1999, 57, 107.

[22] Cinnsealach, R., Boschloo, G., Rao, S.N., Fitzmaurice, D. Sol. Energy Mater. Sol. Cells, 1998, 55, 215.

[23] Gaballah, S.T.; Netzel, T.L. Nucleosides Nucleotides Nucleic Acids, 2002, 21, 681 .

[24] Gaballah, S.T.; Kerr, C.E.; Eaton, B.E.; Netzel, T.L. Nucleosides, Nucleotides Nucleic Acids, 2002, 21, 547.

[25] Ikeda, H.; Fuji, K.; Tanaka, K.; Iso, Y.; Yoneda, F. Chem Pharm. Bull., 1999, 47, 1455.

[26] Connolly, B.A. Nucleic Acids Res., 1987, 15, 3131.

[27] Ocampo, S. M.; Albericio, F.; Fernández, I.; Vilaseca, M.; Eritja, R. Org. Lett., 2005, 7, 4349.

[28] Zaramella, S.; Yeheskiely, E.; Strömberg, R. J. Am. Chem. Soc., 2004, 126, 14029.

[29] Kojima, N.; Sugino, M.; Mikami, A.; Nonaka, K.; Fujinawa, Y.; Ueda, Y.; Sato, K.; Ohtsuka, E.; Matsubara, K.; Komatsu, Y. Nucleic Acids Symp. Ser., 2005, 49,181.

[30] Komatsu, Y.; Kojima, N.; Sugino, M.; Mikami, A.; Nonaka, K.; Fujinawa, Y.; Sugimoto, T.; Sato, K.; Matsubara, K.; Ohtsuka, E. Bioorg. Med. Chem., 2008, 16, 941.

[31] Guzaev, A.; Manoharan, M. Bioorg. Med. Chem. Lett., 1998, 8, 3671.

[32] Ong, W.; Kaifer, A.E. J. Am. Chem. Soc., 2002, 124, 9358.

[33] Ong, W.; Grindstaff, J.; Sobransingh, D.; Toba, R.; Quintela, J.M.; Peinador; Kaifer, A.E. J. Am. Chem. Soc., 2005, 127, 3353.

[34] Heimer, T.A.; D’Arcangelis, S.T.; Farzad, F.; Stipkala, J.M.; Meyer, G.J. Inorg. Chem., 1996, 35, 5319.

[35] Alvira, M.; Eritja, R. Chem. Biodivers., 2007, 4, 2798. 